ARTICLE

Received 29 Nov 2012 | Accepted 14 Mar 2013 | Published 16 Apr $2013 \quad$ DOl: 10.1038/ncomms2736

\title{
Optical visualization of individual ultralong carbon nanotubes by chemical vapour deposition of titanium dioxide nanoparticles
}

Rufan Zhang ${ }^{1}$, Yingying Zhang ${ }^{2}$, Qiang Zhang ${ }^{1}$, Huanhuan Xie ${ }^{1,2}$, Haidong Wang ${ }^{3}$, Jingqi Nie ${ }^{1}$, Qian Wen ${ }^{1} \&$ Fei Wei ${ }^{1}$

Direct visualization and manipulation of individual carbon nanotubes in ambient conditions is of great significance for their characterizations and applications. However, the observation of individual carbon nanotubes usually requires electron microscopes under high vacuum. Optical microscopes are much more convenient to be used, yet their resolution is low. Here we realize the visualization and manipulation of individual ultralong carbon nanotubes under optical microscopes by deposition of $\mathrm{TiO}_{2}$ nanoparticles on them. The strong scattering of $\mathrm{TiO}_{2}$ nanoparticles to visible light renders them visible by optical microscopes. Micro-Ramanspectroscopy measurement of individual carbon nanotubes is greatly facilitated by their optical visualization. With the assistance of $\mathrm{TiO}_{2}$ nanoparticles, individual carbon nanotubes can be easily manipulated under an optical microscope at macroscopic scale and in ambient conditions. Based on our approach, various manipulation of ultralong carbon nanotubes, including cutting, transfer, fabrication of structures/devices and pulling out inner shells of multiwalled carbon nanotubes, are demonstrated.

\footnotetext{
${ }^{1}$ Department of Chemical Engineering, Beijing Key Laboratory of Green Chemical Reaction Engineering and Technology, Tsinghua University, Beijing 100084, China. ${ }^{2}$ Center for Nano and Micro Mechanics, Tsinghua University, Beijing 100084, China. ${ }^{3}$ Key Laboratory for Thermal Science and Power Engineering of Ministry of Education, School of Aerospace, Tsinghua University, Beijing 100084, China. Correspondence and requests for materials should be addressed to Y.Y.Z. (email: yingyingzhang@tsinghua.edu.cn) or to F.W. (email: wf-dce@tsinghua.edu.cn).
} 
$\mathrm{T}$ he advance of material science always benefits from the development of modern characterization or operation techniques. Carbon nanotubes (CNTs) are undoubtedly one of the most attractive materials in the past two decades because of their superior properties and potential applications in many fields. Particularly, ultralong CNTs, with their perfect onedimensional nanostructures and excellent properties, such as extraordinary mechanical strength, ultrahigh electron mobility and excellent electrical conductivity ${ }^{1-3}$, are promising building blocks for high-performance fibres and nanodevices. However, the direct visualization, location and manipulation of individual CNTs, especially single or few-walled CNTs, is extremely difficult because of their nanosized diameters. Advanced analytical equipment, such as scanning electron microscope (SEM), transmission electron microscope (TEM) and atomic force microscope (AFM) and so on, has been employed to characterize the location, morphology and structure of CNTs. The in-situ manipulation of individual CNTs with the help of the above equipment has also been realized ${ }^{4-8}$. However, all the above-mentioned techniques cannot guarantee the efficient location and manipulation of ultralong CNTs, because of their characteristics such as limited accessibility, requiring high vacuum, narrow field of view and/or small operating space.

Optical microscopes are routine facilities in many laboratories. Combing with the open-operation space of optical microscopes, if individual CNTs can be directly observed with an optical microscope, the in-situ observation and manipulation of ultralong CNTs will become much easier. However, the resolution of optical microscopes is much lower than that of SEM, TEM and AFM. Recently, various methods were proposed to 'see' CNTs optically, such as near-field Raman spectroscopy ${ }^{9}$ and fluorescence microscopy ${ }^{10-14}$. However, the preparation of the samples and the identification of individual CNTs are quite complex and timeconsuming by these techniques. Besides, an electro-deposition process of $\mathrm{Ag}$ nanoparticles $(\mathrm{NPs})^{15}$ and a seeded electroless deposition process of $\mathrm{Au} \mathrm{NPs}{ }^{16}$ have been used to visualize individual CNTs on substrates under an optical microscope. Compared with CNTs sitting on substrates, suspended CNTs possess the pristine properties of CNTs and are convenient to be manipulated. However, the above strategies cannot be applied on suspended CNTs because of the fact that these processes are conducted in liquid phases that will destroy the suspended CNTs. Therefore, to explore the full potential of CNTs towards their future applications, a facile and nondestructive visualization method for individual suspended CNTs, especially for those with length up to several centimeters, is in great demand.

In this contribution, a facile and effective method to visualize individual suspended ultralong CNTs by deposition of $\mathrm{TiO}_{2} \mathrm{NPs}$ in a vapour phase is proposed. The strong scattering of $\mathrm{TiO}_{2} \mathrm{NPs}$ to visible light enables the CNTs to be easily observed under optical microscopes even at low magnification, which allows the direct manipulation of individual CNTs, even ultralong ones with length up to centimeters, under optical microscopes. Based on that, we demonstrate various manipulations of individual ultralong CNTs under an optical microscope.

\section{Results}

Deposition of $\mathrm{TiO}_{2}$ NPs on individual ultralong CNTs. The process of depositing $\mathrm{TiO}_{2}$ NPs on ultralong CNTs is illustrated in Fig. 1a-d and Supplementary Fig. S1. Suspended ultralong CNTs were grown by chemical vapour deposition ${ }^{1,17,18}$ on a silicon substrate containing many trenches $(0.5-1 \mathrm{~mm}$ in width and $0.5 \mathrm{~mm}$ in depth). The as-grown CNTs were brought into contact with $\mathrm{TiCl}_{4}$ vapour for in-situ deposition of $\mathrm{TiO}_{2} \mathrm{NPs}_{\text {s }}$ (see Methods section for details).
After the suspended CNTs contacted $\mathrm{TiCl}_{4}$ for $1-7 \mathrm{~s}$, many $\mathrm{TiO}_{2}$ NPs deposited on the CNTs. Figure 1e-h show the necklacelike $\mathrm{CNT} \mathrm{TiO} 2$ hybrids. Most of the large $\mathrm{TiO}_{2} \mathrm{NPs}$ have spheric morphologies with diameters of $100-1,000 \mathrm{~nm}$. Most of these spherical $\mathrm{TiO}_{2} \mathrm{NPs}$ completely wrap the suspended CNTs, forming coaxial structures (Fig. 1f-h). In contrast, other particles, especially those with small diameters $(20-100 \mathrm{~nm})$, usually adhere to the outer wall of suspended CNTs (Fig. 1e,f). Small $\mathrm{TiO}_{2} \mathrm{NPs}$ are distributed between adjacent large $\mathrm{TiO}_{2} \mathrm{NPs}$. The interspace of the small NPs usually ranges from 20 to $1000 \mathrm{~nm}$, whereas the interspace of larger NPs usually ranges from 1 to $5 \mu \mathrm{m}$. Pristine CNT segments can be clearly observed between the particles. The ultralong CNT shown in Fig. If is a triple-walled CNT with a diameter of $3.5 \mathrm{~nm}$ (see the inset in Fig. 1f).

Compared with the depostion methods of $\mathrm{TiO}_{2} \mathrm{NPs}$ on CNTs reported previously ${ }^{19-25}$, the vapour phase-directed deposition reported here is easy to be conducted and does not require the CNT surfaces to be pre-modified. Although a series of covalent and non-covalent methods have been reported to deposit metal ${ }^{26-30}$ or metal oxide NPs ${ }^{19-25,30-42}$ on CNTs, most of these methods require the CNTs to be functionalized or modified with surfactants 39,42 , and the processes are conducted in liquid phases $^{17-39,41,42}$. These strategies cannot be applied on individual suspended ultralong CNTs because of the fact that they will destroy the suspended CNTs. Besides, atomic layer deposition ${ }^{43}$, which avoids the use of liquid phases, has been used to conformably coat CNTs sitting on substrates ${ }^{41,43}$ or pre-functionalized suspended $\mathrm{CNTs}^{44}$ with metal oxide films. In comparison, we realized the deposition of $\mathrm{TiO}_{2}$ NPs on as-prepared suspended CNTs in a vapour phase. $\mathrm{TiCl}_{4}$, which is a highly volatile metal halide, was

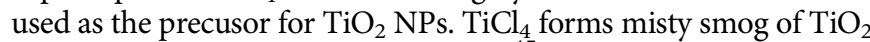
and $\mathrm{HCl}$ upon contact with humid air ${ }^{45}$. When $\mathrm{TiCl}_{4}$ vapour gets into contact with suspended CNTs, tiny $\mathrm{TiO}_{2}$ NPs nucleate on the CNTs (Fig. 1b). The suspended CNTs have a role of templates in tailoring the size of $\mathrm{TiO}_{2} \mathrm{NPs}^{35}$. The $\mathrm{TiO}_{2} \mathrm{NPs}^{4}$ are hydrophilic ${ }^{46}$, whereas the pristine CNTs are hydrophobic ${ }^{47}$. As a result, the following formed $\mathrm{TiO}_{2}$ in the vapour phase prefers to deposite on the preformed $\mathrm{TiO}_{2}$ nucleus. The $-\mathrm{OH}$ groups on the surface of $\mathrm{TiO}_{2}$ NPs also catalyse $\mathrm{TiCl}_{4}$ hydrolysis ${ }^{48}$, resulting in the vapour phase epitaxial growth of the preformed $\mathrm{TiO}_{2} \mathrm{NPs}$. The small $\mathrm{TiO}_{2}$ NPs attached on the outer wall of the suspended CNTs gradually grow large and wrap the CNTs with the continuously growth, forming coaxial strucutures. Meanwhile, a large amount of newly nucleated $\mathrm{TiO}_{2} \mathrm{NPs}$ formed and adhered to the suspended CNTs. Therefore, small and large $\mathrm{TiO}_{2}$ NPs coexist on the suspended CNTs. The interactions between the $\mathrm{TiO}_{2} \mathrm{NPs}$ and the CNTs is dominated by van der Waals force 22,35 .

The contact time of CNTs with the $\mathrm{TiCl}_{4}$ vapour and the air humidity have important roles in determining the diameter and number distribution of the $\mathrm{TiO}_{2} \mathrm{NPs}$ deposited on the suspended CNTs as reported previously 22 . As shown in Supplementary Fig. S2, the diameter and number of the $\mathrm{TiO}_{2} \mathrm{NPs}$ are proportional with the contact time and the relative air humidity. The size of the $\mathrm{TiO}_{2}$ NPs on CNTs obeys a typical logarithmic normal distribution as shown in Supplementary Fig. S2a. Supplementary Table S1 shows the diameter and interspace distribution of $\mathrm{TiO}_{2} \mathrm{NPs}$ with different contact time. With the increase of contact time, the mean diameter of $\mathrm{TiO}_{2} \mathrm{NPs}(\mu)$ increases, whereas the particle interspace decreases (Supplementary Fig. S2b). No matter what the interspace is, the variance of the interspace is $<0.1 \mu \mathrm{m}^{2}$, indicating that the interspace of the $\mathrm{TiO}_{2} \mathrm{NPs}$ is uniform. The air humidity affects the hydrolysis rate of $\mathrm{TiCl}_{4}$. The higher the air humidity, the larger the hydrolysis rate is. As a result, $\mu$ increases with the increase of air humidity (Supplementary Fig. S2c). When the relative air humidity is $>70 \%$, the increasing trend of $\mu$ gradually slows down because of the hydrolysis rate reaching its limit. 
a

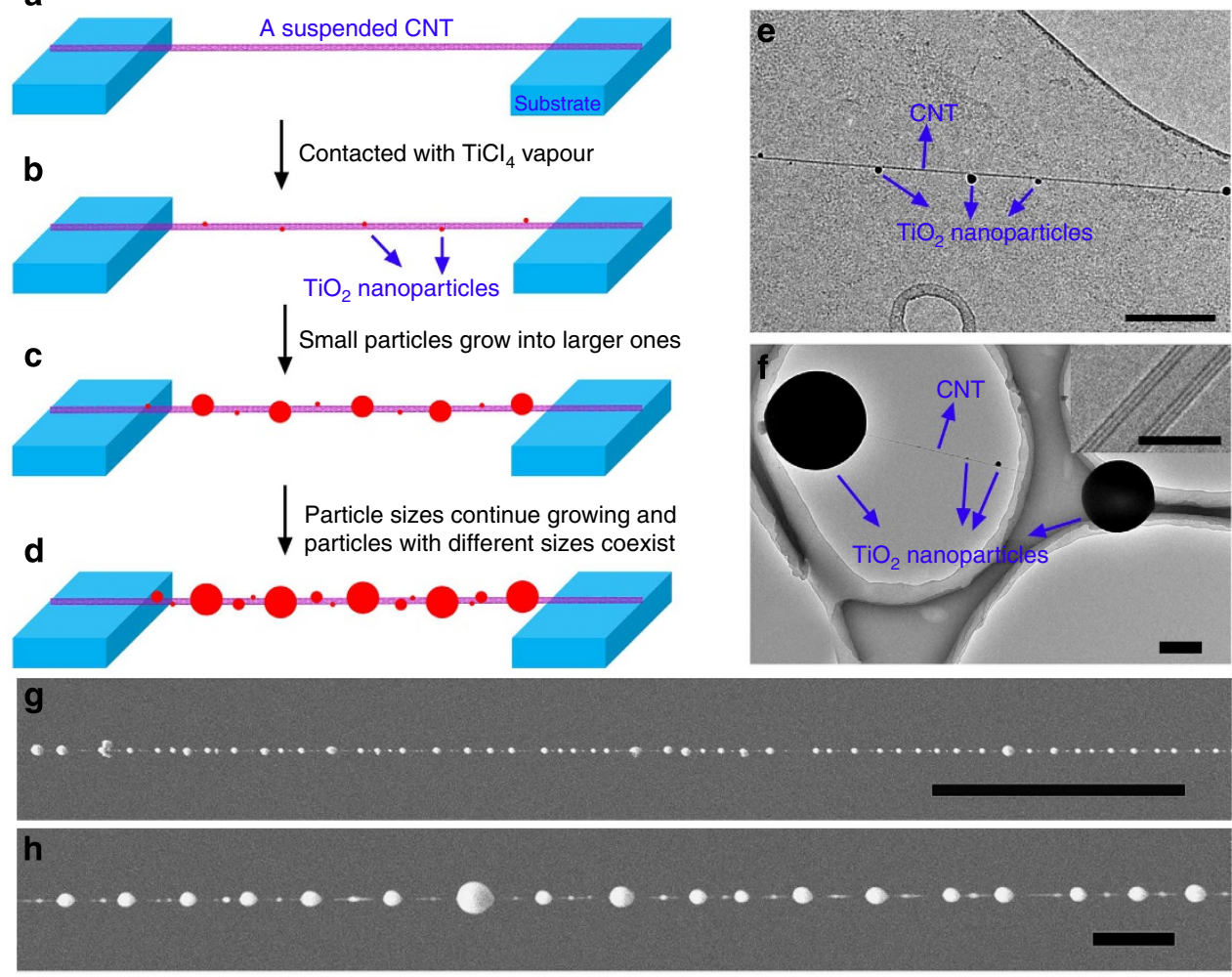

Figure 1 | Deposition of $\mathrm{TiO}_{\mathbf{2}}$ NPs on ultralong CNTs. (a-d) Schematic illustration for depositing $\mathrm{TiO}_{2}$ NPs on suspended CNTs. (e) TEM images of CNTs with small $\mathrm{TiO}_{2}$ NPs prepared with contact time of 0.1-0.3 s. Scale bar, $200 \mathrm{~nm}$. (f) TEM image of CNT with TiO $2 \mathrm{NPs}$ with various sizes prepared with contact time of $7.0 \mathrm{~s}$. Scale bar, $500 \mathrm{~nm}$. Inset: high-resolution TEM image of the CNT shown in panel $\mathbf{f}$. Scale bar, $5 \mathrm{~nm}$. (g,h) SEM images of suspended CNTs decorated with $\mathrm{TiO}_{2}$ NPs prepared with contact time of 5.0 s. Scale bar, $10 \mu \mathrm{m}$ (in $\mathbf{g}$ ); $2 \mu \mathrm{m}$ (in $\mathbf{h}$ ).

Optical visualization of individual ultralong CNTs. The diameters of most $\mathrm{TiO}_{2}$ NPs $(100-1,000 \mathrm{~nm})$ are comparative with the wavelength of visible light $(300-700 \mathrm{~nm})$, rendering the $\mathrm{TiO}_{2}$ NPs a strong Mie-scattering ability ${ }^{49-51}$. The CNTs decorated with $\mathrm{TiO}_{2}$ NPs can be easily observed and tracked under an optical microscope. Figure 2 shows typical optical images of suspended CNTs decorated with $\mathrm{TiO}_{2} \mathrm{NPs}$. We can easily locate and track the suspended CNTs with the aid of $\mathrm{TiO}_{2} \mathrm{NPs}$. Even when the suspended CNTs with $\mathrm{TiO}_{2}$ NPs are broken and finally fall down onto the substrate, they can still be clearly observed (shown by the red arrow in Fig. 2a). No matter for what incident light intensity, the $\mathrm{TiO}_{2}$ NPs always have a strong scattering ability to the incident light (Fig. 2a-c), especially in the dark field mode (Fig. 2b). The suspended CNTs decorated with $\mathrm{TiO}_{2} \mathrm{NPs}$ can be clearly observed even with a very low magnification $(\times 50)$ of microscopes (Fig. 2d,e). Particularly, $\mathrm{TiO}_{2} \mathrm{NPs}$ as small as $100 \mathrm{~nm}$ (Fig. 2f, shown by arrow ' 3 ') are still visible under the optical microscope. Furthermore, the $\mathrm{TiO}_{2} \mathrm{NPs}$ can be removed through an acid-washing method to facilate their futher characterizations and applications (Supplementary Fig. S3).

\section{Discussion}

Micro-Raman spectra measurement of individual CNTs is greatly facilitated by the optical visualization of individual CNTs. With the assistance of $\mathrm{TiO}_{2} \mathrm{NPs}$, the position and orientation of the ultralong suspended CNTs can be easily identified under the optical microscope of micro-Raman-spectroscopy equipment. The laser spot can be focused on the pristine CNTs between two adjacent $\mathrm{TiO}_{2}$ NPs (Fig. 3a,b), where strong Raman scattering signals are usually obtained. The deposition of $\mathrm{TiO}_{2} \mathrm{NPs}$ does not influence the Raman spectra of CNTs. Figure $3 \mathrm{c}$ shows the
Raman G-band of a CNT before and after deposition of $\mathrm{TiO}_{2}$ NPs. The Raman spectra measured with and without $\mathrm{TiO}_{2} \mathrm{NPs}$ were almost the same (see Supplementary Discussion and Supplementary Fig. S4).

Furthermore, the visualization of individual CNTs under optical microscopes enabled by the deposition of $\mathrm{TiO}_{2} \mathrm{NPs}$ greatly facilitates the manipulation of individual CNTs in macro scale and ambient conditions. For example, with the assistance of $\mathrm{TiO}_{2}$ NPs, ultralong CNTs can be cut and the as-obtained CNT segments can be used as samples for further characterization. Figure $4 \mathrm{a}$ shows a process of cutting and transferring a CNT segment. The cut-off CNT segments can be used for structural or property measurements, such as micro-Raman spectra measurement, TEM characterization and electrical measurement, facilitating the characterization of ultralong CNTs. Moreover, the CNT segments can be employed to fabricate complicated $\mathrm{CNT}$ architectures. Figure $4 \mathrm{~b}$ shows a process to fabricate a CNT cross-structure, which can be completed within several minutes. More complicated CNT architectures could be fabricated in the same way. Note that all the above procedures were carried out under an optical microscope, which provides an open space for the manipulation of individual CNTs.

The optical visualization of individual CNTs also facilitates the design and fabrication of various devices. Figures $5 \mathrm{a}, \mathrm{b}$ show that a suspended CNT can be bent by a gas flow, which can be used to monitor external gas flows. Accordingly, given a known gas velocity, this phenomenon can be used to measure the tensile strength and Young's modulus of the suspended CNTs. Figure $5 \mathrm{c}, \mathrm{d}$ show that a suspended $\mathrm{CNT} / \mathrm{TiO}_{2}$ chain oscillates under a sound wave, which can be monitored by an optical microscope. This phenomenon can be used to test the fatigue life of the suspended CNTs. Besides, an external electric field can also 

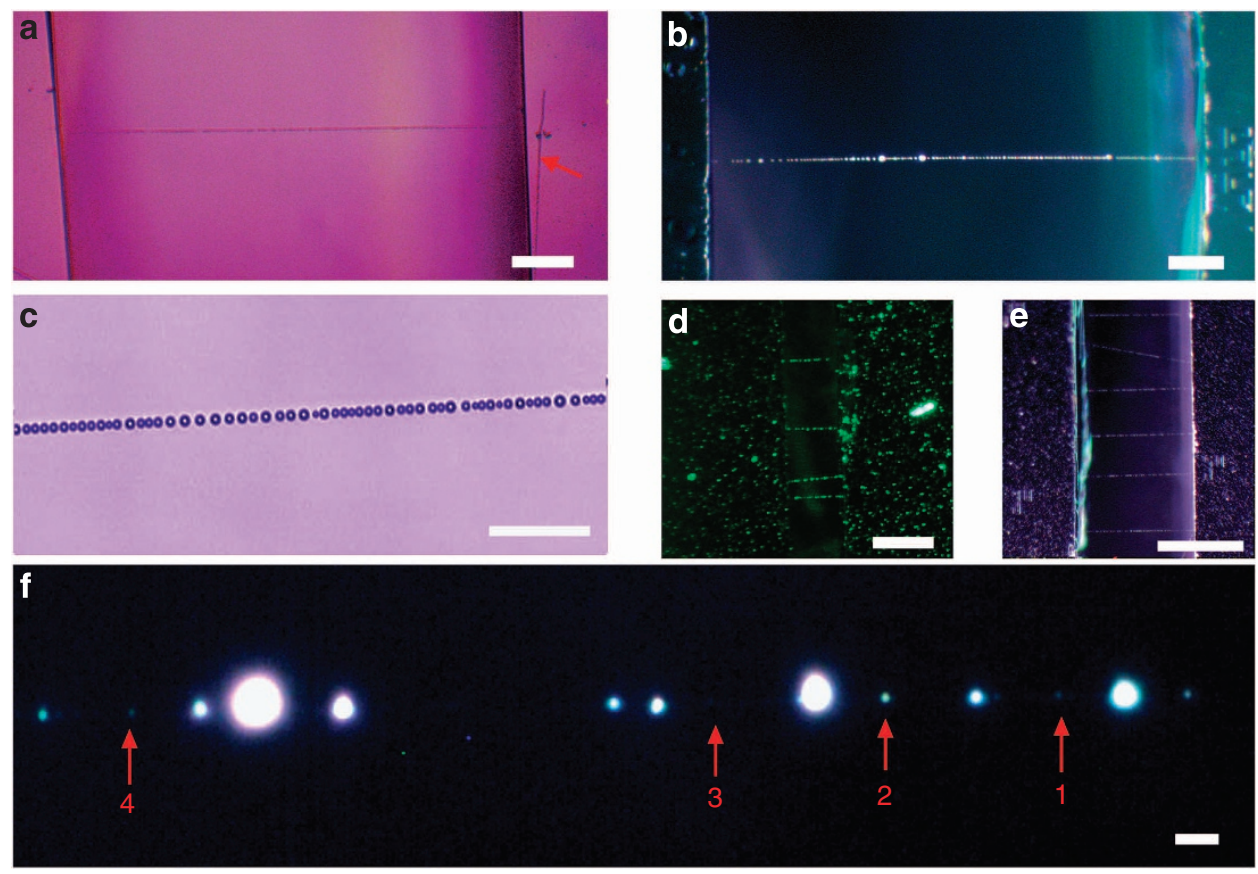

Figure 2 | Optical images of suspended CNTs. $(\mathbf{a}, \mathbf{c})$ Bright field modes. The red arrow in panel a shows a broken CNT falling on the substrate. Scale bar, $100 \mu \mathrm{m}$ (in a); $20 \mu \mathrm{m}$ (in c). (b) Dark field mode. Scale bar, $100 \mu \mathrm{m}$. (d,e) Optical images with low magnification ( $\times 50$ ). Scale bar, $1 \mathrm{~mm}$. The light source in panel $\mathbf{d}$ is green laser, whereas the light source in panel $\mathbf{e}$ is visible light. (f) High-resolution optical image of a suspended $\mathrm{CNT}_{\text {decorated }}$ with TiO ${ }_{2} \mathrm{NPs}_{\text {. }}$ The small $\mathrm{TiO}_{2}$ NPs indicated by the red arrows have diameters $<200 \mathrm{~nm}$. Scale bar, $1 \mu \mathrm{m}$.

a
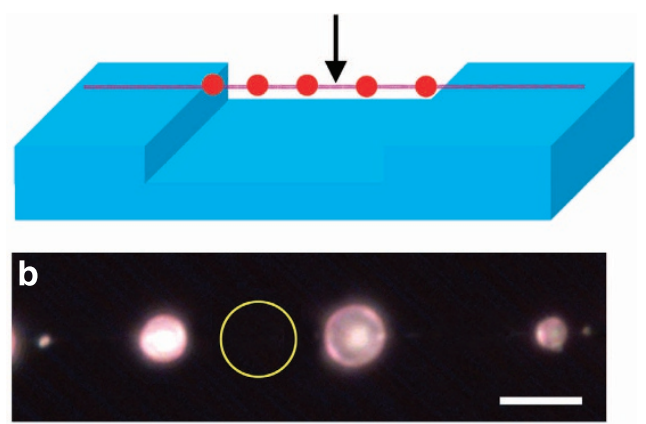

c

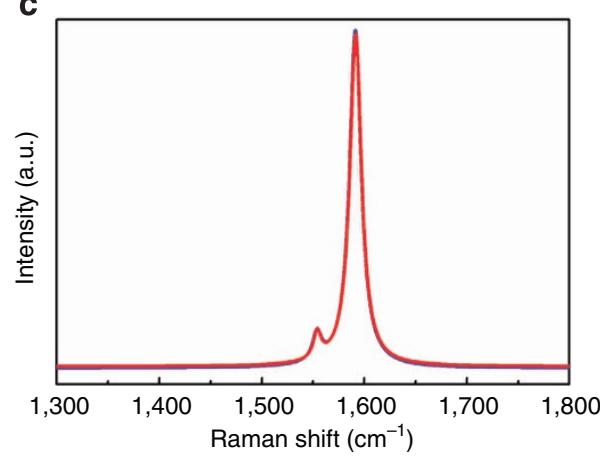

Figure 3 | $\mathrm{TiO}_{\mathbf{2}}$ NP-assisted micro-Raman spectra measurement of a suspended CNT. (a) Illustration for micro-Raman spectra measurement. (b) Optical image showing the position of laser spot (yellow circle) on the suspended CNTs for micro-Raman spectra measurement. Scale bar, $1 \mu \mathrm{m}$. (c) Raman spectra of the same CNT before (the red line) and after (the blue line) deposition of $\mathrm{TiO}_{2} \mathrm{NPs}$.

be used to manipulate suspended $\mathrm{CNT} / \mathrm{TiO}_{2}$ chain, exhibiting interesting behaviours (Supplementary Fig. S5). Similarly, more devices based on individual CNTs could be fabricated and more properties of individual CNTs could be measured with the $\mathrm{TiO}_{2}$ NP-assisted optical visualization of CNTs.

Moreover, with the aid of $\mathrm{TiO}_{2} \mathrm{NPs}$, the intershell sliding behaviours of ultralong multiwalled CNTs can be tracked and investigated in macroscopic scale under an optical microscope. Figure 6a shows a suspended double-walled CNT (DWCNT) (see its high-resolution TEM image in the upper inset in Fig. 6e) and a probe for manipulation. With the probe approaching and finally contacting with the CNT, the CNT firmly fixed to the probe due to the strong van der Waals force between them (Fig. 6b) (binders such as silver paste could be used for a better fixation effect). Then, the suspended CNT could be stretched by moving the probe in a certain direction. The $\mathrm{TiO}_{2}$ NPs deposited on the suspended CNT are excellent markers to locate the outer shell of the CNT. During the process, the interspace of two adjacent NPs (shown by two red arrows in Fig. 6c) suddenly had an increase, indicating that the outer shell of the DWCNT was broken. Although the pulled-out inner shell could not be observed directly under an optical microscope, its location and length could be determined from the broken ends of the outer shell. Figure 6d-e shows that the inner shell of the suspended DWCNT had been continuously pulled out, resulting in a $3.43-\mathrm{mm}$-long singlewalled CNT. Note that this is the longest inner tube pulled out from a multiwalled CNT, at least two orders of magnitude longer than that of previous reported results ${ }^{52}$. Moreover, if we fix a force cantilever onto the probe, the external force needed for pulling out the inner shell of the CNT and the friction between shells of the CNT could be measured. The tensile strength, breaking strain of the CNTs, could also be obtained.

In conclusion, the optical visualization of individual CNTs was realized by deposition of $\mathrm{TiO}_{2}$ NPs on suspended ultralong CNTs in a vapour phase. $\mathrm{TiO}_{2}$ NPs were uniformly deposited on suspended CNTs using $\mathrm{TiCl}_{4}$ as the precursor, forming 
a
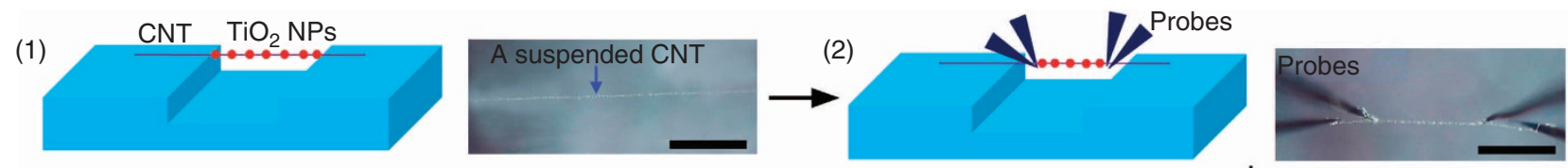

(4)

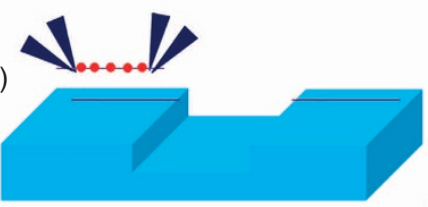

(5)

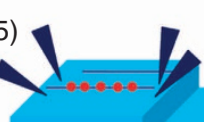

$\downarrow$

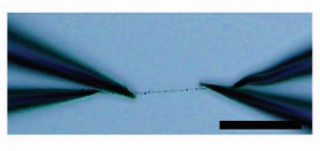

(3)

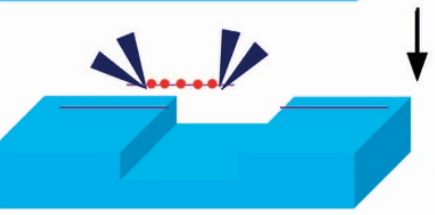

$\downarrow$

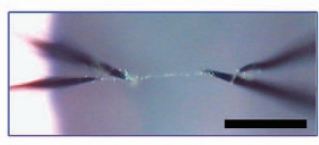

(6)
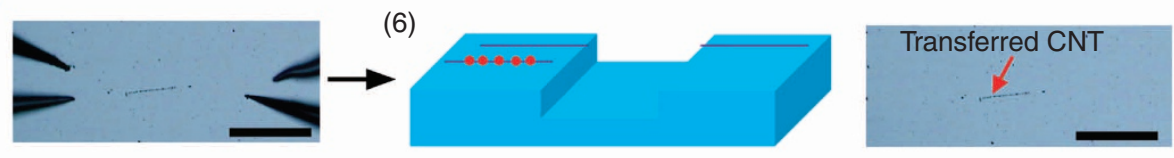

b
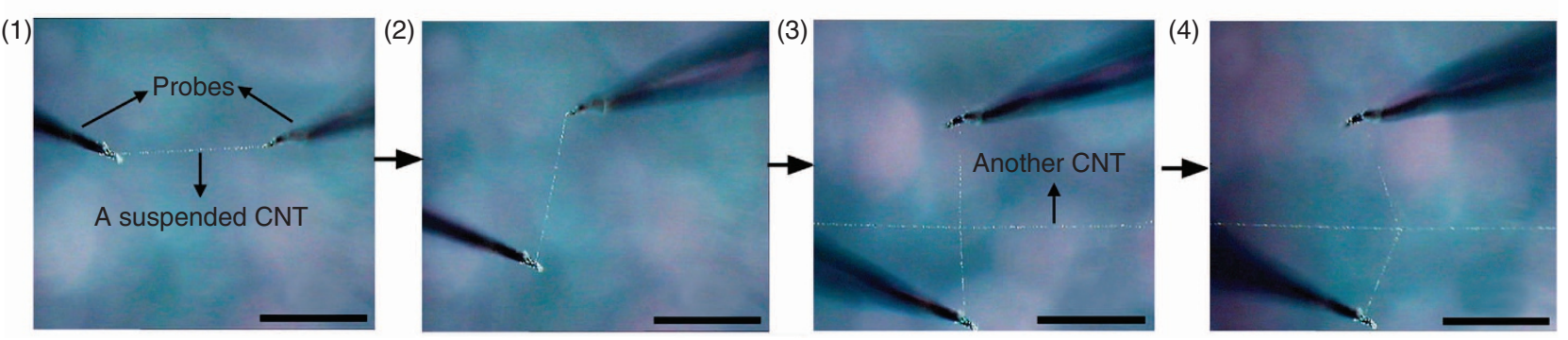

Figure 4 | Cutting and transferring of individual CNTs and fabrication of a CNT cross-structure. (a) Cutting and transferring of individual CNTs (left: schematic illustration; right: optical images). (1) A suspended CNT with $\mathrm{TiO}_{2}$ NPs. Scale bar, $50 \mu \mathrm{m}$. (2) Four probes were moved onto the CNT to cut a CNT segment. Scale bar, $50 \mu \mathrm{m}$. (3-6) A CNT segment (ca. $70 \mu \mathrm{m}$ ) was cut off from the CNT. Scale bar, $50 \mu \mathrm{m}$. Then the probes served as tweezers to transfer the CNT segment. (b) Cutting a CNT segment and fabricating a CNT cross-structure. (1) Cutting a CNT segment from a suspended CNT using two probes. Scale bar, $50 \mu \mathrm{m}$. (2) Changing the position and orientation of the CNT segment. Scale bar, $50 \mu \mathrm{m}$. (3) Moving the CNT segment onto another CNT and fabricating a CNT cross-structure. Scale bar, $50 \mu \mathrm{m}$. (4) Deforming the CNT cross-structure by moving the probes. Scale bar, $50 \mu \mathrm{m}$.
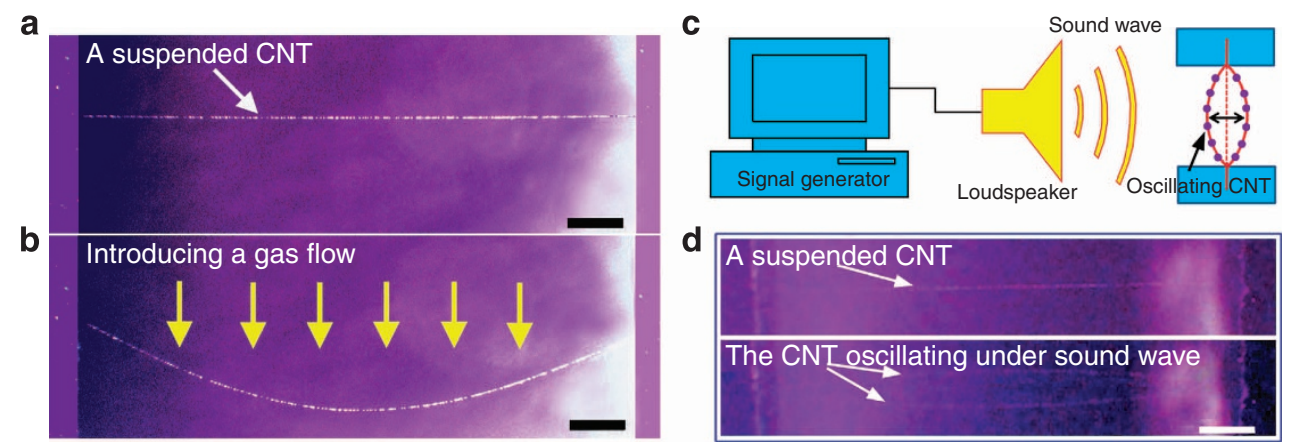

Figure 5 | Gas-flow-induced bending and sound-wave-induced oscillation of suspended CNTs. (a) A suspended CNT. Scale bar, $100 \mu$ m. (b) Gas-flowinduced bending of the CNT. Scale bar, $100 \mu \mathrm{m}$. (c) Illustration of oscillating a suspended CNT with sound wave. (d) Snapshot images of an oscillating CNT under sound wave. Scale bar, $100 \mu \mathrm{m}$.

necklace-like CNT $\mathrm{TiO}_{2}$ hybrids. With the strong scattering to visible light, the $\mathrm{TiO}_{2}$ NPs could be easily observed under optical microscopes, enabling the facile location and tracking individual CNTs. Mico-Raman spectra measurement was greatly facilitated by the optical visualization of individual CNTs. Besides, with the assistance of $\mathrm{TiO}_{2}$ NPs, direct manipulation of individual ultralong CNTs in macro scale and under ambient conditions could be easily carried out. We showed that, individual CNTs could be cut, transferred and fabricated into structures/devices. Moreover, we demonstrated the pulling out of an inner shell with a length up to $3.43 \mathrm{~mm}$ from a DWCNT under an optical microscope. Our results indicate that the $\mathrm{TiO}_{2} \mathrm{NP}$-assisted optical visualization of individual CNTs greatly facilitates the characterization and manipulation of individual CNTs, and opens opportunities to explore macro-scale properties of individual
CNTs. Note that the NP-assisted optical visualization of CNTs could be easily applied on other nanomaterials, especially onedimensional, suspended ones. More studies related to the macroscale mechanical properties of individual ultralong CNTs based on this technique are under way in our laboratory.

\section{Methods}

Deposition of $\mathrm{TiO}_{2}$ NPs on CNTs. The deposition process was conducted at room temperature in a fume hood, where there was a humidity controller to regulate and measure the relative air humidity (Supplementary Fig. $\mathrm{S} 1$ ). $\mathrm{TiCl}_{4}$ liquid was controllably introduced into a beaker. $\mathrm{The}^{\mathrm{TiCl}}{ }_{4}$ liquid immediately formed misty smog of $\mathrm{TiO}_{2}$ and $\mathrm{HCl}$ upon contact with humid air. The substrates with suspended CNTs were taken into contact with $\mathrm{TiCl}_{4}$ vapour and $\mathrm{TiO}_{2}$ smog for deposition of $\mathrm{TiO}_{2} \mathrm{NPs}$. The contact time usually ranged from 3.0 to $7.0 \mathrm{~s}$. After that, the substrates with suspended CNTs were taken away to terminate the deposition process. 

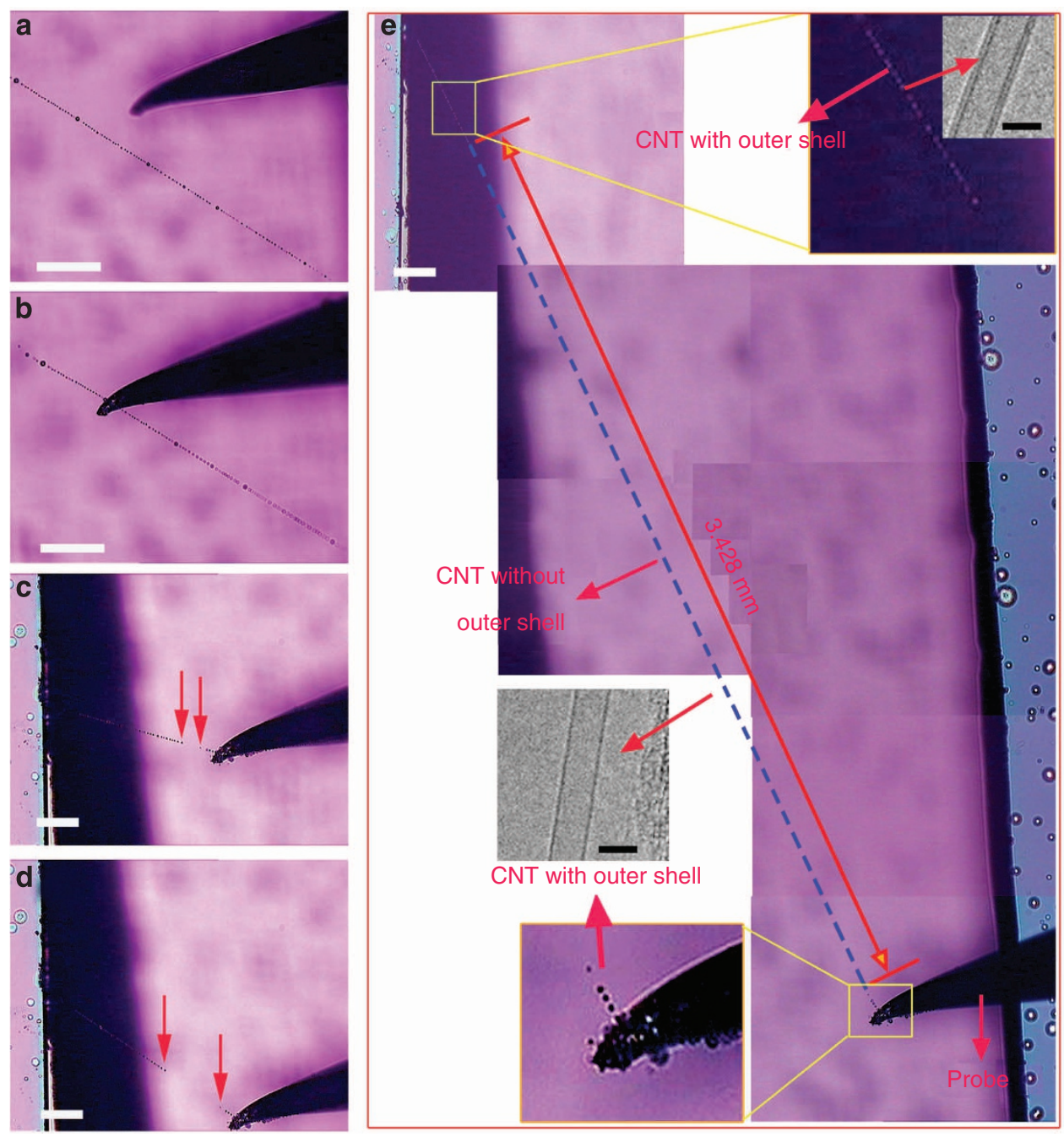

Figure 6 | Pulling out the inner tube from a DWCNT. (a,b) Approaching a probe to a DWCNT and then contacting it. Scale bar, $100 \mu \mathrm{m}$. (c,d) Stretching the DWCNT and then pulling out its inner shell. Scale bar, $200 \mu \mathrm{m}$. (e) A 3.43-mm-long singlewalled CNT being pulled out from the DWCNT. Scale bar, $20 \mu \mathrm{m} ; 2 \mathrm{~nm}$ (lower inset); $2 \mathrm{~nm}$ (upper inset).

Characterization. The CNTs were characterized by SEM (JSM 7401F, $1.0 \mathrm{kV}$ ), high-resolution TEM (JEM 2010, $120.0 \mathrm{kV}$ ) and Raman spectroscopy (Horiba HR $800,632.8 \mathrm{~nm}$ ). An optical microscope (long working distance metallography microscope, FS-70Z) was used for the optical characterization and manipulation of individual CNTs.

Cutting and transferring CNT segments. The substrate with suspended CNTs was put on the stage of an optical microscope. Four probes were fixed on two pedestals on the microscope framework. The probes could be moved precisely in X, $\mathrm{Y}$ and $\mathrm{Z}$ directions by wheeling the knobs fixed on the pedestals. The four probes were taken into contact with the suspended CNTs, forming two scissor-like structures to cut the CNT. After a CNT segment was cut off, the probes then served as tweezers to transfer the CNT segment.

Pulling out the inner tube from an individual DWCNT. When the suspended DWCNTs decorated with $\mathrm{TiO}_{2}$ NPs were placed under an optical microscope, the probes were taken into contact with the suspended DWCNTs. Then, the DWCNT was stretched by moving the probes in a certain direction. The deformation of the DWCNT could be directly monitored under the optical microscope. AFM cantilevers could be fixed onto the probes by silver paste to measure the forces applied on the DWCNTs.

Gas-flow-induced bending of suspended CNTs. A steady air flow was introduced through a glass tube $(5 \mathrm{~cm}$ in length and $1 \mathrm{~mm}$ in diameter) to blow the suspended CNTs decorated with $\mathrm{TiO}_{2}$ NPs. The fixed ends of the CNTs remained fixed when the suspended part was stretched. The elongation of the suspended CNTs was recorded by a digital camera (Carnon, SX500 IS). The gas velocity of the air flow was controlled by a mass flow metre (Sevenstar, CS200A 100SCC).
Sound-wave-induced oscillation of suspended CNTs. The suspended $\mathrm{CNT} / \mathrm{TiO}_{2}$ hybrids could be excited to oscillate by a sound wave from a loudspeaker linked to a signal generator (Tektronix, AFG3000C). The oscillation of the suspended CNTs was recorded by a digital camera (Carnon, SX500 IS).

Manipulation of suspended CNTs in an electric field. A minus voltage was applied on a suspended CNT, and a probe (with positive voltage) was used to attract the suspended CNT (Supplementary Fig. S5a). The suspended CNT is served as an electric field sensor and shows a quick response. Another example is shown in Supplementary Fig. S5b; a 60- $\mu \mathrm{m}$ long free-drooping CNT was fixed on a metal probe with another probe placed $120 \mu \mathrm{m}$ away. When a voltage was applied between the two probes (left: $2 \mathrm{~V}$; right: $-2 \mathrm{~V}$ ), the suspended CNT was immediately attracted to the left probe (Supplementary Fig. S5b (2)). Once the voltage was removed, the CNT immediately fell down back to a drooping state (Supplementary Fig. S5b (3)). However, when an equal voltage was applied between the two probes (for example, $2 \mathrm{~V}$ for both probes), the strong electric repulsion between the two probes immediately repelled the freely drooping CNT away to the right side (Supplementary Fig. S5b (4)). Once the voltage between the two probes was removed, the repelled CNT became drooping again. If the voltage difference between the two probes was applied again, the CNT would be immediately attracted to the left probe.

Removing of the $\mathrm{TiO}_{\mathbf{2}} \mathbf{N P s}$ from CNT. A substrate with many $\mathrm{TiO}_{2} \mathrm{NPs}$ and short CNTs was put into a beaker containing $20 \mathrm{ml}$ sulphuric acid solution (concentration $65 \%$ ). $\left(\mathrm{NH}_{4}\right)_{2} \mathrm{SO}_{4}$ powder of $8 \mathrm{~g}$ was added into the sulphuric acid solution as a dissolving promoter. Then the solution was heated to boil for $2 \mathrm{~h}$ Then the substrate out was taken out and the $\mathrm{TiO}_{2} \mathrm{NPs}$ could be completely removed. 


\section{References}

1. Zhang, R. F. et al. Superstrong ultralong carbon nanotubes for mechanical energy storage. Adv. Mater. 23, 3387-3391 (2011).

2. Wen, Q. et al. $100 \mathrm{~mm}$ long, semiconducting triple-walled carbon nanotubes Adv. Mater. 22, 1867-1871 (2010).

3. Wang, X. S. et al. Fabrication of ultralong and electrically uniform single-walled carbon nanotubes on clean substrates. Nano Lett. 9, 3137-3141 (2009).

4. Thelander, C. \& Samuelson, L. AFM manipulation of carbon nanotubes: realization of ultra-fine nanoelectrodes. Nanotechnology 13, 108-113 (2002).

5. Demczyk, B. G. et al. Direct mechanical measurement of the tensile strength and elastic modulus of multiwalled carbon nanotubes. Mater. Sci. Eng. A 334, 173-178 (2002).

6. Postma, H. W. C., Sellmeijer, A. \& Dekker, C. Manipulation and imaging of individual single-walled carbon nanotubes with an atomic force microscope. Adv. Mater. 12, 1299-1302 (2000)

7. Yu, M. F. et al. Strength and breaking mechanism of multiwalled carbon nanotubes under tensile load. Science 287, 637-640 (2000)

8. Yu, M. F. et al. Three-dimensional manipulation of carbon nanotubes under a scanning electron microscope. Nanotechnology 10, 244-252 (1999).

9. Anderson, N., Hartschuh, A. \& Novotny, L. Chirality changes in carbon nanotubes studied with near-field Raman spectroscopy. Nano Lett. 7, 577-582 (2007).

10. Duggal, R. \& Pasquali, M. Dynamics of individual single-walled carbon nanotubes in water by real-time visualization. Phys. Rev. Lett. 96, 246104 (2006).

11. Chaudhary, S., Kim, J. H., Singh, K. V. \& Ozkan, M. Fluorescence microscopy visualization of single-walled carbon nanotubes using semiconductor nanocrystals. Nano Lett. 4, 2415-2419 (2004).

12. Didenko, V. V., Moore, V. C., Baskin, D. S. \& Smalley, R. E. Visualization of individual single-walled carbon nanotubes by fluorescent polymer wrapping. Nano Lett. 5, 1563-1567 (2005).

13. Otobe, K. et al. Fluorescence visualization of carbon nanotubes by modification with silicon-based polymer. Nano Lett. 2, 1157-1160 (2002).

14. Tsyboulski, D. A., Bachilo, S. M. \& Weisman, R. B. Versatile visualization of individual single-walled carbon nanotubes with near-infrared fluorescence microscopy. Nano Lett. 5, 975-979 (2005).

15. Huang, S. et al. Identification of the structures of superlong oriented singlewalled carbon nanotube arrays by electrodeposition of metal and Raman spectroscopy. J. Am. Chem. Soc. 130, 11860-11861 (2008).

16. Chu, H., Cui, R., Wang, J., Yang, J. \& Li, Y. Visualization of individual singlewalled carbon nanotubes under an optical microscope as a result of decoration with gold nanoparticles. Carbon 49, 1182-1188 (2011)

17. Wen, Q. et al. Growing $20 \mathrm{~cm}$ long DWNTs/TWNTs at a rapid growth rate of

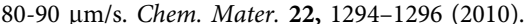

18. Huang, S. M., Woodson, M., Smalley, R. \& Liu, J. Growth mechanism of oriented long single walled carbon nanotubes using "fast-heating" chemical vapour deposition process. Nano Lett. 4, 1025-1028 (2004).

19. Llobet, E. et al. Carbon nanotube- $\mathrm{TiO}_{2}$ hybrid films for detecting traces of $\mathrm{O}_{2}$. Nanotechnology 19, 375501 (2008)

20. Eder, D. \& Windle, A. H. Carbon-inorganic hybrid materials: the carbonnanotube/ $\mathrm{TiO}_{2}$ interface. Adv. Mater. 20, 1787-1793 (2008).

21. Darányi, M. et al. Layer-by-layer assembly of $\mathrm{TiO}_{2}$ nanowire/carbon nanotube films and characterization of their photocatalytic activity. Nanotechnology 22, 195701 (2011).

22. Eder, D. \& Windle, A. H. Morphology control of $\mathrm{CNT}^{-\mathrm{TiO}_{2}}$ hybrid materials and rutile nanotubes. J. Mater. Chem. 18, 2036-2043 (2008).

23. Zhao, Y. et al. Super-long aligned $\mathrm{TiO}_{2} /$ carbon nanotube arrays. Nanotechnology 21, 505702 (2010).

24. Mishra, A., Banerjee, S., Mohapatra, S. K., Graeve, O. A. \& Misra, M. Synthesis of carbon nanotube- $\mathrm{TiO}_{2}$ nanotubular material for reversible hydrogen storage. Nanotechnology 19, 445607 (2008).

25. Liang, Y., Wang, H., Sanchez Casalongue, H., Chen, Z. \& Dai, H. TiO nanocrystals grown on graphene as advanced photocatalytic hybrid materials. Nano Res. 3, 701-705 (2010).

26. Aksel, S. \& Eder, D. Catalytic effect of metal oxides on the oxidation resistance in carbon nanotube-inorganic hybrids. J. Mater. Chem. 20, 9149-9154 (2010).

27. Guldi, D. M. et al. CNT-CdTe versatile donor-acceptor nanohybrids. J. Am. Chem. Soc. 128, 2315-2323 (2006)

28. Li, H. et al. A general and efficient route to fabricate carbon nanotube-metal nanoparticles and carbon nanotube-inorganic oxides hybrids. Adv. Funct. Mater. 20, 3864-3873 (2010).

29. Tang, H. et al. High dispersion and electrocatalytic properties of platinum on well-aligned carbon nanotube arrays. Carbon 42, 191-197 (2004).

30. Lee, Y., Song, H. J., Shin, H. S., Shin, H. J. \& Choi, H. C. Spontaneous formation of transition-metal nanoparticles on single-walled carbon nanotubes anchored with conjugated molecules. Small 1, 975-979 (2005).

31. Eder, D. Carbon nanotube-inorganic hybrids. Chem. Rev. 110, 1348-1385 (2010).
32. Tasis, D., Tagmatarchis, N., Bianco, A. \& Prato, M. Chemistry of carbon nanotubes. Chem. Rev. 106, 1105-1136 (2006).

33. Han, W. Q. \& Zettl, A. Coating single-walled carbon nanotubes with tin oxide. Nano Lett. 3, 681-683 (2003).

34. Krissanasaeranee, M., Wongkasemjit, S., Cheetham, A. K. \& Eder, D. Complex carbon nanotube-inorganic hybrid materials as next-generation photocatalysts. Chem. Phys. Lett. 496, 133-138 (2010).

35. Georgakilas, V. et al. Decorating carbon nanotubes with metal or semiconductor nanoparticles. J. Mater. Chem. 17, 2679-2694 (2007).

36. Li, H., Ha, C. S. \& Kim, I. Fabrication of carbon nanotube $/ \mathrm{SiO}_{2}$ and carbon nanotube/ $\mathrm{SiO}_{2} / \mathrm{Ag}$ nanoparticles hybrids by using plasma treatment. Nano. Res. Lett. 4, 1384-1388 (2009).

37. Neocleus, S., Pattinson, S., Motta, A. M. M., Windle, A. \& Eder, D. Hierarchical carbon nanotube-inorganic hybrid structures involving CNT arrays and CNT fibres. Funct. Mater. Lett. 4, 83-89 (2011).

38. Shan, Y. \& Gao, L. Multiwalled carbon nanotubes $/ \mathrm{Co}_{3} \mathrm{O}_{4}$ nanocomposites and its electrochemical performance in lithium storage. Chem. Lett. 33, 1560-1561 (2004)

39. Li, X., Qin, Y., Picraux, S. \& Guo, Z. X. Noncovalent assembly of carbon nanotube-inorganic hybrids. J. Mater. Chem. 21, 7527-7547 (2011).

40. Zhan, Y. et al. A novel carbon nanotubes $/ \mathrm{Fe}_{3} \mathrm{O}_{4}$ inorganic hybrid material: synthesis, characterization and microwave electromagnetic properties. J. Magn Magn. Mater. 323, 1006-1010 (2011).

41. Javey, A. et al. Self-aligned ballistic molecular transistors and electrically parallel nanotube arrays. Nano Lett. 4, 1319-1322 (2004).

42. Holzinger, M. et al. Sidewall functionalization of carbon nanotubes. Angew. Chem. Int. Ed. 40, 4002-4005 (2001).

43. Hausmann, D. M., Kim, E., Becker, J. \& Gordon, R. G. Atomic layer deposition of hafnium and zirconium oxides using metal amide precursors. Chem. Mater. 14, 4350-4358 (2002).

44. Farmer, D. B. \& Gordon, R. G. Atomic layer deposition on suspended single walled carbon nanotubes via gas-phase noncovalent functionalization. Nano Lett. 6, 699-703 (2006)

45. Rigo, M., Canu, P., Angelin, L. \& Della Valle, G. Kinetics of TiCl4 hydrolysis in a moist atmosphere. Ind. Eng. Chem. Res. 37, 1189-1195 (1998).

46. Sakai, N., Wang, R., Fujishima, A., Watanabe, T. \& Hashimoto, K. Effect of ultrasonic treatment on highly hydrophilic $\mathrm{TiO} 2$ surfaces. Langmuir 14, 5918-5920 (1998).

47. Hummer, G., Rasaiah, J. C. \& Noworyta, J. P. Water conduction through the hydrophobic channel of a carbon nanotube. Nature 414, 188-190 (2001).

48. Li, Y., Fan, Y. \& Chen, Y. A novel method for preparation of nanocrystalline rutile $\mathrm{TiO} 2$ powders by liquid hydrolysis of TiCl4. J. Mater. Chem. 12, 1387-1390 (2002).

49. Mishchenko, M. I., Travis, L. D. \& Lacis, A. A. Scattering, Absorption, and Emission of Light by Small Particles (Cambridge university press, 2002)

50. Kerker, M. The Scattering of Light (Academic Press, New York, 1969).

51. Huber, E. \& Frost, M. Light scattering by small particles. Aqua 47, 87-94 (1998).

52. Hong, B. H. et al. Extracting subnanometer single shells from ultralong multiwalled carbon nanotubes. Proc. Natl Acad. Sci. USA 102, 14155-14158 (2005)

\section{Acknowledgements}

The work is supported by the Foundation for the National Basic Research Program of China (2011CB932602 and 2013CB934200).

\section{Author contributions}

F.W. proposed and supervised the project. R.Z. designed and performed the experiments and wrote the manuscript. Y.Y.Z. co-supervised the project and designed the outline of the manuscript. Q.Z. and Q.W. participated in data analysis and the manuscript preparation. H.X. participated in the synthesis of ultralong CNTs. H.W. conducted the Raman characterization. J.N. helped with the TEM characterization.

\section{Additional information}

Supplementary Information accompanies this paper at http://www.nature.com/ naturecommunications

Competing financial interests: The authors declare no competing financial interests.

Reprints and permission information is available online at http://npg.nature.com/ reprintsandpermissions/

How to cite this article: Zhang, R. et al. Optical visualization of individual ultralong carbon nanotubes by chemical vapour deposition of titanium dioxide nanoparticles. Nat. Commun. 4:1727 doi: 10.1038/ncomms2736 (2013). 